\title{
Deciphering the Genetic Basis of Lodging Resistance in Wild Rice Oryza longistaminata
}

\author{
Weixiong Long, Dong Dan, Zhengqing Yuan, Yunping Chen, Jie Jin, Weilong Yang, \\ Zhihong Zhang, Nengwu Li and Shaoqing Li*
}

State Key Laboratory of Hybrid Rice, Key Laboratory for Research and Utilization of Heterosis in Indica Rice of Ministry of Agriculture, Engineering Research Center for Plant Biotechnology and Germplasm Utilization of Ministry of Education, College of Life Science, Wuhan University, Wuhan, China

OPEN ACCESS

Edited by: Petr Smýkal,

Palacký University, Czechia

Reviewed by:

Robert Henry,

University of Queensland, Australia Keisuke Nagai,

Nagoya University, Japan

*Correspondence:

Shaoqing Li

shaoqingli@whu.edu.cn

Specialty section:

This article was submitted to

Plant Breeding,

a section of the journal

Frontiers in Plant Science

Received: 03 March 2020

Accepted: 23 April 2020

Published: 29 May 2020

Citation:

Long W, Dan D, Yuan Z, Chen Y, Jin J, Yang W, Zhang Z, Li N and LiS (2020) Deciphering the Genetic Basis of Lodging Resistance in Wild Rice

Oryza longistaminata.

Front. Plant Sci. 11:628.

doi: 10.3389/fpls.2020.00628
The abuse of fertilizer results in tall rice plants that are susceptible to lodging and reduced plant yield. Hence, it is important to identify and utilize the quantitative trait loci (QTLs)/genes for lodging resistance breeding. Oryza longistaminata exhibits a strong stem and high biomass productivity, which could be a candidate gene pool for cultivars lodging resistance improvement. Here, a set of $152 \mathrm{BC}_{2} \mathrm{~F}_{20}$ lines derived from a cross between a cultivated line 93-11 and O. longistaminata was evaluated for lodging resistance. QTL mapping analysis combined with single-nucleotide polymorphism (SNP) marker derived from high-throughput sequencing identified 12 QTLs for stem diameter (SD), 11 QTLs for stem length (SL), and 3 QTLs for breaking strength (BS). Of which, 14 QTLs were first identified from O. longistaminata. A major QTL, $q L R 1$, which was delimited to a region $\sim 80 \mathrm{~kb}$ on chromosome 1, increased stem diameter, stem length, and breaking strength. Another major QTL, $q L R 8$, that was delimited in an interval $\sim 120 \mathrm{~kb}$ on chromosome 8, significantly enhanced the breaking strength. These results provide evidence that $O$. longistaminata can be exploited to develop lodging-resistant rice lines.

Keywords: lodging resistance, QTLs, Oryza longistaminata, wild rice, stem diameter

\section{INTRODUCTION}

Lodging in cereal crops is a major problem that results in decreased grain yield and deteriorated grain quality (Berry et al., 2004). Development of lodging-resistant varieties to cope with this challenge has been widely attended to increase yield in rice, maize, and other crops (Zhu et al., 2013; Peng et al., 2014; Yadav et al., 2017). Plant breeders have reduced lodging risk by introducing the semi-dwarf gene $s d 1$, known as the "Green Revolution Gene" (Peng et al., 1999). However, recent studies showed that semi-dwarf trait in rice limits photosynthesis and biomass production leading to a yield penalty (Murai et al., 2002). Additionally, it may have a negative pleiotropic effect on culm morphology (Okuno et al., 2014). In other words, the gibberellin synthesis gene ( $s d 1)$ widely applied in Green Revolution rice also reduces the culm strength by decreasing culm diameter, which

Abbreviations: BIL, backcross inbred line; BS, breaking strength; HI, harvest index; HN, Hainan; LR, lodging resistance; PVE, phenotypic variation explained; QTL, quantitative traits loci; SD, stem diameter; SL, stem length; WH, Wuhan. 
TABLE 1 | Performance of lodging associated trait in backcross inbred lines (BILs) within 2 years.

\begin{tabular}{|c|c|c|c|c|c|c|c|}
\hline \multirow[t]{2}{*}{ Traits } & \multirow[t]{2}{*}{ Environment } & \multirow[t]{2}{*}{ Year } & \multicolumn{5}{|c|}{ BILs } \\
\hline & & & Min & Max & Average & SD & CV (\%) \\
\hline \multirow[t]{4}{*}{ Stem diameter (mm) } & WH & 2017 & 5.27 & 12.78 & 7.94 & 1.17 & 14.74 \\
\hline & & 2018 & 5.17 & 13.25 & 7.92 & 1.22 & 15.40 \\
\hline & $\mathrm{HN}$ & 2017 & 5.79 & 12.23 & 8.66 & 1.17 & 13.51 \\
\hline & & 2018 & 5.52 & 13.00 & 8.68 & 1.24 & 14.29 \\
\hline \multirow[t]{4}{*}{ Stem length (cm) } & WH & 2017 & 64.1 & 178.67 & 109.24 & 31.54 & 28.87 \\
\hline & & 2018 & 78.6 & 191.53 & 119.70 & 40.64 & 33.95 \\
\hline & $\mathrm{HN}$ & 2017 & 41.08 & 157.90 & 83.59 & 29.54 & 35.34 \\
\hline & & 2018 & 49.11 & 181.67 & 103.02 & 30.62 & 29.72 \\
\hline \multirow[t]{4}{*}{ Breaking strength (N) } & $\mathrm{HN}$ & 2017 & 5.81 & 29.68 & 14.95 & 4.64 & 31.04 \\
\hline & & 2018 & 6.13 & 30.28 & 15.12 & 4.65 & 30.75 \\
\hline & WH & 2017 & 5.41 & 30.86 & 15.50 & 4.80 & 30.97 \\
\hline & & 2018 & 5.42 & 31.33 & 15.51 & 4.84 & 31.21 \\
\hline
\end{tabular}

WH and HN represent Wuhan and Hainan, respectively.
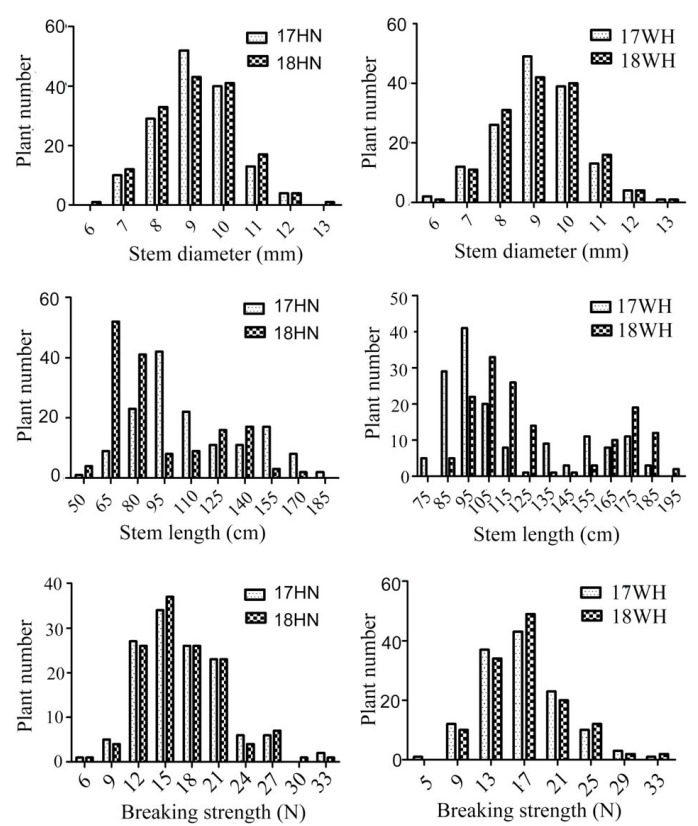

FIGURE 1 | Phenotyping of lodging-resistant traits of 152 Oryza longistaminata backcross inbred lines (BILs). WH and HN present Wuhan and Hainan, respectively.

makes it difficult to further improve lodging resistance by one semi-dwarf genes alone. Thus, it is important to search for alien genes favorable for breeding lodging-resistant rice.

Lodging resistance is a complex quantitative trait, which is affected by many factors, such as culm morphology, culm diameter and length, cellulose content, and environment conditions. Previous studies have shown that the culm diameter and size are highly correlated with the lodging resistance of rice (Kashiwagi and Ishimaru, 2004; Kashiwagi et al., 2006; Fan et al., 2018; Sowadan et al., 2018). Ookawa et al. (2010) identified four QTLs for bending moment at breaking and section modulus of the fourth internodes derived from two indica rice varieties with strong culms. Similarly, Kashiwagi et al. (2008) detected five QTLs for pushing resistance from a backcross between Nipponbare and Kasalath. Shailesh Yadav et al. (2017) mapped 12 QTLs for lodging resistance from a backcross between Swarna and Moroberekan. Moreover, three functional genes including prl5, SCM2, and SCM3 have been identified to regulate stem diameter. Interestingly, SCM2 increased not only culm strength but also spikelet number. However, few reports have been made about lodging resistance in wild rice apart from three QTLs for culm-base thickness derived from pLIA1, which carried Oryza longistaminata's chromosome segments (Gichuhi et al., 2016). Hence, exploitation of new QTLs or genes from wild rice contributing to lodging resistance will help enrich the gene pool for improvement of lodging resistance in rice.

The perennial wild species $O$. longistaminata, which showed large stem diameter, thick stem wall, and high biomass production, is believed to help improve rice lodging resistance (Khush, 1997). In our study, we conducted QTL analysis of potential lodging resistance of $O$. longistaminata by evaluation of stem diameter, stem length, and breaking strength using an advanced backcross inbred line (BIL) population derived from a cross between $O$. longistaminata and 93-11. Totally, 26 QTLs for lodging resistance were detected, of which 12 QTLs for stem diameter (SD), 11 QTLs for stem length (SL), and three QTLs for breaking strength (BS) were derived from O. longistaminata. These novel QTLs will lay the foundation for breeding strong lodging resistant rice and broaden our understanding of the genetic basis of rice lodging resistance.

\section{MATERIALS AND METHODS}

\section{Plant Materials and Field Experiments}

The recurrent indica rice variety 93-11, wild rice O. longistaminata, and 152 backcross inbred lines (BILs) 


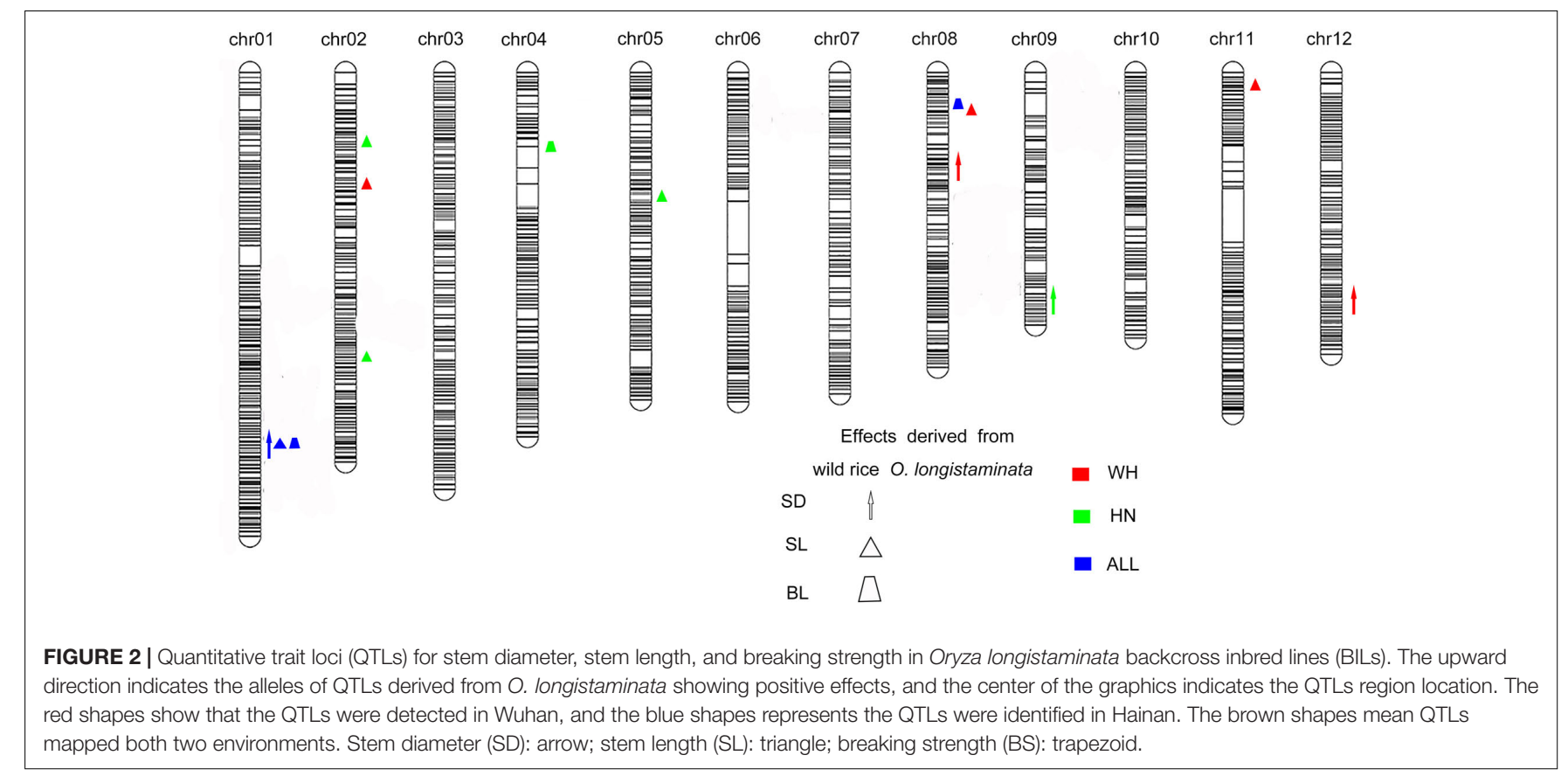

derived from a cross between them were used in this research. Field experiments were conducted in Linshui, Hainan province during the rice-growing season from late October to late March and in Wuhan, Hubei province during mid-May to late-September in 2017 and 2018. Each BIL was planted in five rows with 10 plants in each row at a spacing of $20 \mathrm{~cm} \times 16.5 \mathrm{~cm}$. A randomized complete block design with three replications was used in each experiment.

\section{Measurement of Lodging \\ Resistance-Related Traits}

At 2 weeks after heading, five plants of each BIL and 93-11 were selected to investigate the lodging resistance traits. The stem diameter (SD) of the fifth internode from the top of each selected plant was measured using an electronic Vernier caliper in the field. Stem length of the internode (SL) from the top was measured from the ground to the base of the panicle. The breaking strength (BS), a parameter for the physical strength of the stem, was measured at the last internode of the plants using a plant lodging tester (YYD-1A, Zhejiang TOP Instrument Co., Ltd., China).

\section{Statistical Analysis}

All the data were analyzed using SPSS 20.0 statistical software. Mean values of each trait were used for further QTL analysis. The standard deviation of the means was calculated using Microsoft Excel software. Correlations between the lodging resistance traits were evaluated using Pearson's correlation.

\section{QTL Analysis and Sequence Analysis}

A total of 2,432 bin markers was used to construct the genetic linkage map covering the whole genome as described by Jin et al. (2018). Inclusive composite interval mapping combined with additive mapping (ICIM-ADD) method was used to detect more precise lodging resistance QTLs (Meng et al., 2015). The significant logarithm of odds (LOD) value threshold for each trait was determined following the $5 \%$ permutation test with 1,000 replicates. The putative genes on the QTL region were identified based on the RIGW ${ }^{1}$.

\section{RESULTS}

\section{Phenotypic Evaluation of Lodging Resistance-Related Traits}

Significant differences for the three measured traits were found between the two parents in both trials (Supplementary Figure 1 and Table 1). The wild rice performed better than 93-11 for all of the three lodging-related traits, indicating that O. longistaminata can efficiently improve the lodging resistance of rice.

For the BIL population, the variation of stem diameter, stem length, and breaking strength in Hainan ranged from 5.79 to $12.23 \mathrm{~mm}, 41.08$ to $157.90 \mathrm{~cm}$, and 5.81 to $29.68 \mathrm{~N}$, respectively, with an average of $8.66 \mathrm{~mm}, 83.59 \mathrm{~cm}$, and $14.95 \mathrm{~N}$, respectively, in 2017 (Figure 1). Correspondingly, the coefficient of variation $(\mathrm{CV})$ of stem diameter (SD), stem length (SL), and breaking length (BS) was 13.51, 35.34, and 31.04\%, respectively (Table 1). In 2018 at Hainan, the variation of SD, SL, and $\mathrm{BS}$ of $O$. longistaminata BIL population ranged from 5.52 to $13.00 \mathrm{~mm}, 49.11$ to $181.67 \mathrm{~cm}$, and 6.13 to $30.28 \mathrm{~N}$, with an average of $8.68 \mathrm{~mm}, 103.03 \mathrm{~cm}$, and $15.12 \mathrm{~N}$, respectively. Correspondingly, the CV of SD, SL, and BS was 14.29, 29.72, and $30.75 \%$, respectively.

\footnotetext{
${ }^{1}$ http://rice.hzau.edu.cn/rice/
} 
TABLE 2 | Quantitative trait loci (QTLs) of lodging resistance derived from Oryza longistaminata in Hainan.

\begin{tabular}{|c|c|c|c|c|c|c|c|c|c|c|c|c|c|}
\hline \multirow[t]{2}{*}{ Investigated traits } & \multirow[t]{2}{*}{ QTLs } & \multirow[t]{2}{*}{ Chr } & \multirow[t]{2}{*}{ Pos (cM) } & \multirow[t]{2}{*}{ L/Bin } & \multirow[t]{2}{*}{ R/Bin } & \multirow[t]{2}{*}{ L/bp } & \multirow[t]{2}{*}{$R / b p$} & \multicolumn{2}{|c|}{ LOD } & \multicolumn{2}{|c|}{ PVE } & \multicolumn{2}{|c|}{ Add } \\
\hline & & & & & & & & 2017 & 2018 & 2017 & 2018 & 2017 & 2018 \\
\hline \multirow[t]{2}{*}{ Stem diameter } & qSD1.1 & 1 & 226 & $1-171$ & $1-172$ & 35964905_36016970 & 35557085_35961004 & 5.78 & & 13.68 & & 0.49 & \\
\hline & qSD9.1 & 9 & 8 & $9-6$ & $9-8$ & 20987075_21077941 & 20967923_20984846 & 3.17 & 4.99 & 6.87 & 10.85 & 0.63 & 0.80 \\
\hline \multirow[t]{3}{*}{ Stem length } & qSL1.1 & 1 & 216 & $1-159$ & $1-160$ & 36587534_36864307 & 36569506_36587533 & 31.22 & 39.27 & 59.40 & 67.03 & 25.55 & 25.87 \\
\hline & qSL2.1 & 2 & 145 & $2-201$ & $2-202$ & 8863323_8895438 & 8841866_8863322 & 4.58 & & 5.53 & & 9.28 & \\
\hline & qSL2.2 & 2 & 119 & $2-247$ & $2-148$ & 18779567_18968062 & 18713595_18770186 & & 3.77 & & 10.22 & & 2.57 \\
\hline \multirow[t]{3}{*}{ Breaking strength } & qBS1.1 & 1 & 216 & $1-161$ & $1-162$ & 36539238_36569505 & 36515725_36536296 & 4.87 & 5.07 & 13.70 & 16.76 & 1.86 & 2.06 \\
\hline & qBS4.1 & 4 & 120 & $4-97$ & $4-98$ & 19770490_20005045 & 19663208_19770489 & 2.66 & & 7.10 & & 2.14 & \\
\hline & qBS8.1 & 8 & 32 & $8-32$ & $8-33$ & 8075498_8118334 & 8118335_8196568 & 3.30 & & 8.70 & & 2.21 & \\
\hline
\end{tabular}

TABLE 3 | Quantitative trait loci (QTLs) associated with lodging resistance derived from Oryza longistaminata identified in a population of backcross inbred lines (BILs) evaluated in 2017 and 2018 in Wuhan and Hainan, China.

\begin{tabular}{|c|c|c|c|c|c|c|c|c|c|c|c|c|c|}
\hline \multirow[t]{2}{*}{ Investigated traits } & \multirow[t]{2}{*}{ QTLs } & \multirow[t]{2}{*}{ Chr } & \multirow[t]{2}{*}{ Pos (cM) } & \multirow[t]{2}{*}{ L/Bin } & \multirow[t]{2}{*}{ R/Bin } & \multirow[t]{2}{*}{ L/bp } & \multirow[t]{2}{*}{$R / b p$} & \multicolumn{2}{|c|}{ LOD } & \multicolumn{2}{|c|}{ PVE } & \multicolumn{2}{|c|}{ Add } \\
\hline & & & & & & & & 2017 & 2018 & 2017 & 2018 & 2017 & 2018 \\
\hline \multirow[t]{3}{*}{ Stem diameter } & qSD8.1 & 8 & 74 & $8-59$ & $8-60$ & 9870144_9978376 & 20727323_21034035 & 3.43 & 4.55 & 18.83 & 8.03 & 1.12 & 1.13 \\
\hline & qSD1.1 & 1 & 216 & $1-161$ & $1-162$ & 36539238_36569505 & 36515725_36536296 & & 13.29 & & 25.37 & & 0.65 \\
\hline & qSD12.1 & 12 & 156 & $12-168$ & $12-169$ & 22817923_22863684 & 22863685_22983852 & & 51.39 & & 34.80 & & 1.99 \\
\hline \multirow[t]{5}{*}{ Stem length } & qSL1.1 & 1 & 216 & $1-160$ & $1-161$ & 36587534_36864307 & 36569506_36587533 & 2.77 & 53.00 & 7.21 & 79.89 & 9.11 & 31.64 \\
\hline & qSL1.2 & 1 & 286 & $1-310$ & $1-311$ & 23526094_23553497 & 23494864_23526093 & 3.43 & & 9.12 & & 10.51 & \\
\hline & qSL2.3 & 2 & 189 & $2-252$ & $2-253$ & 10475930_10505727 & 10520137_10562305 & 2.70 & & 8.09 & & 22.59 & \\
\hline & qSL8.1 & 8 & 78 & $8-59$ & $8-60$ & 9870144_9978376 & 20727323_21034035 & & 3.32 & & 4.42 & & 18.61 \\
\hline & qSL11.2 & 11 & 290 & $11-128$ & $11-129$ & 1_370955 & 1355747_137440 & & 2.50 & & 5.01 & & 23.36 \\
\hline \multirow[t]{2}{*}{ Breaking strength } & qBS1.1 & 1 & 216 & $1-161$ & $1-162$ & 36539238_36569505 & 36515725_36536296 & & 4.02 & & 11.22 & & 1.75 \\
\hline & qBS8.1 & 8 & 32 & $8-31$ & $8-32$ & 8075498_8118334 & 8118335_8196568 & & 3.78 & & 10.22 & & 2.57 \\
\hline
\end{tabular}


In Wuhan, the SD ranged from 5.27 to $12.78 \mathrm{~mm}$ and 5.17 to $13.25 \mathrm{~cm}$, with the CV of 14.73 and $15.40 \%$ in 2017 and 2018, respectively. The SL ranged from 64.1 to $178.67 \mathrm{~cm}$, and 78.60 to $191.53 \mathrm{~cm}$, with the CV of 28.87 and $33.95 \%$ in 2017 and 2018, respectively. The BS ranged from 5.41 to $30.86 \mathrm{~N}$ and 5.42 to $31.33 \mathrm{~N}$, with the CV of 30.97 and $31.21 \%$ in 2017 and 2018, respectively (Table 1 ). These results indicate that the BS, SL, and $\mathrm{SD}$ of $O$. longistaminata BILs all showed great variation among different lines; BS and SL have more genetic diversity than SD regardless of environments.

\section{Correlations Among Lodging Resistance-Related Traits}

Significant correlations $(P<0.05)$ were found between the traits studied (Supplementary Tables 2, 3). Breaking strength was found to be positively correlated with stem diameter and stem length in Hainan $(P<0.001)$. However, breaking strength was negatively correlated with stem length at Wuhan in 2018 (Supplementary Table 2). The negative correlation may be due to the photoperiod sensitivity of some BILs. These results indicate that wild rice O. longistaminata can improve the lodging resistance without the stem length reduction. In other words, it is possible to breed high yield and high biomass cultivars with high lodging resistance, which may provide appropriate genetic resources for breeding tall super yielding rice (Yuan, 2017).

\section{QTL Mapping of Lodging \\ Resistance-Associated Traits}

A total of 26 QTLs associated with stem diameter, stem length, and breaking strength were detected in the population of BILs (Figure 2). Among these, O. longistaminata supplied the superior allele at 14 QTLs (Tables 2, 3), while at the remaining 12 QTLs, the variety 93-11 supplied the superior alleles (Supplementary Tables 2, 3). Of which, eight QTLs including two for stem diameter, three for stem length, and three for breaking strength were identified from $O$. longistaminata in Hainan during the 2 years. Two QTLs, qSD1.1 and qSD9.1, were detected for stem diameter. The QTL $q$ SD9.1 was detected in two consecutive years with a LOD of 3.17 and 4.99 and a PVE of 6.87 and $10.85 \%$ in 2017 and 2018, respectively. qSD1.1 was identified in 2017, and it explained $13.68 \%$ of the phenotypic variation with a LOD of 5.78. The three QTLs ( $q S L 1.1, q S L 2.1$, and $q S L 2.2)$ contributing to stem length were detected on chromosomes 1 and 2, and positive alleles were contributed by O. longistaminata (Figure 2). The QTL qSL1.1 was detected in both 2017 and 2018, and it explained 59.40 and $67.03 \%$ of the phenotypic variation, respectively. QTLs $q S L 2.1$ and $q S L 2.2$ explained phenotypic variation of 5.53 and $10.22 \%$, respectively. The three QTLs, $q B S 1.1, q B S 4.1$, and $q B S 8.1$, contributing to stem breaking strength were mapped on chromosomes 1, 4, and 7. Among them, QTL qBS1.1 was repeatedly detected across 2 years with the PVE of 5.07 and $1.86 \%$, respectively. While $q B S 4.1$ and $q B S 8.1$ were detected only in 2017, they explained the phenotypic variance of 7.10 and $8.70 \%$, respectively.

A total of 10 QTLs for lodging-related traits were derived from O. longistaminata and were detected in Wuhan. Three
QTLs (qSD1.1, qSD8.1, and $q S D 12.1)$ for stem diameter were detected on chromosomes 1, 8, and 12. The QTL qSD8.1 was consistently detected in 2017 and 2018 with a LOD of 3.43 and 4.55 , with the PVE of 18.33 and $8.03 \%$, respectively. The QTLs $q S D 1.1$ and $q$ SD12.1 were identified in 2018 and explained phenotypic variation of 25.37 and $34.80 \%$, respectively. Five QTLs, qSL1.1, qSL1.2, qSL2.3, qSL8.1, and qSL11.2, which were associated with stem length were mapped on chromosomes 1 , 2, 3, 8, and 11, respectively. The QTL qSL1.1 was detected in both years and explained phenotypic variation of 7.21 and $79.89 \%$, respectively. The QTLs $q S L 1.2$ and $q S L 2.3$ were identified in 2017 and had PVE of 9.12 and 8.09\%, respectively. QTLs qSL8.1 and qSL11.2 were mapped in 2018 with a LOD value of 3.32 and 2.50 and PVE of 4.42 and $5.01 \%$, respectively. Two QTLs controlling breaking strength, qBS1.1 and $q B S 8.1$, were identified in 2018 with PVE of 11.22 and $10.22 \%$, respectively.

\section{Colocalization and Stability of QTLs Associated With Lodging Resistance}

To investigate the genetic effects of the QTLs responsible for lodging resistance, all the QTLs for which O. longistaminata had the superior allele at two sites were further analyzed. The QTLs $q S D 1.1, q S L 1.1$, and $q B S 1.1$ were colocalized on chromosome 1 (Figure 2). Another QTL, qBS8.1, was detected both in Wuhan and Hainan. The QTL hotspot qSD1.1/qSL1.1/qBS1.1 explained $25.37 \%$ of the variance for stem diameter, $79.89 \%$ for stem length, and $16.76 \%$ for breaking strength, which indicates that this locus may play an important role for lodging resistance in rice. What is more, this pleiotropic QTL was first detected in wild rice, and we named it lodging resistance 1 ( $q L R 1$ ). Meanwhile, another QTL, qBS8.1, explained lodging resistance variance for breaking strength of 8.70 and $10.22 \%$ at Hainan and Wuhan, respectively (Tables 2, 3) and has been renamed as lodging resistance 8 (qLR8).

\section{Confirmation of the $q L R 1$ and $q L R 8$ for Lodging Resistance}

To further confirm the function of the newly identified genetic locus of $q L R 1$ and $q L R 8$ in O. longistaminata, a high-resolution mapping with several BILs, including BIL 1708, 1719, 1829, 1738, 1755,1795 , and 1769, was performed. High-resolution mapping of several BILs possessing the superior O. longistaminata allele at qLR1 helped narrow down the QTL to an interval between BIN1161 and BIN1-162 (Figure 3A). Among the BILs, the average breaking strength of the lines with the superior O. longistaminata allele at $q L R 1$ was $19.60 \mathrm{~N}$, which was significantly higher than those without $q L R 1(14.32 \mathrm{~N}, P<0.001)$. Furthermore, the average stem diameter and stem length of the lines carrying qLR1 was $9.72 \mathrm{~mm}$ and $123.12 \mathrm{~cm}$, which were significantly $(P<0.01)$ larger than the lines without $q L R 1$, which had an average stem diameter and stem length of $7.46 \mathrm{~mm}$ and $64.46 \mathrm{~cm}$, respectively (Figure 3B). These results indicate that the $q L R 1$ from $O$. longistaminata can significantly increase lodging resistance. 
A

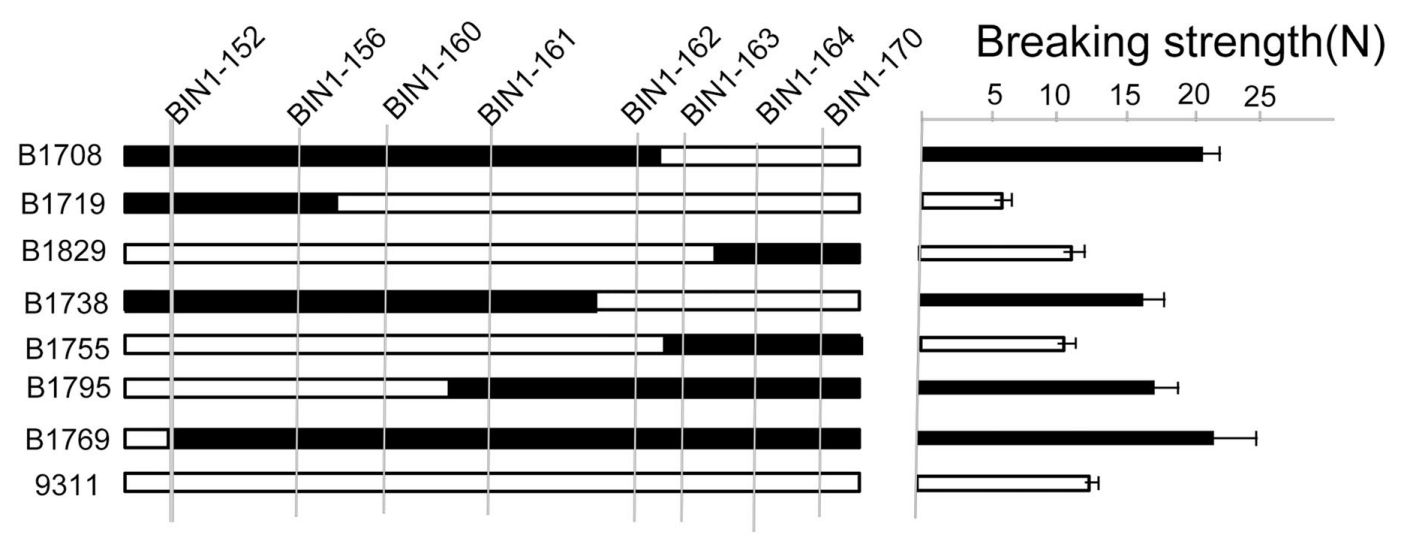

B

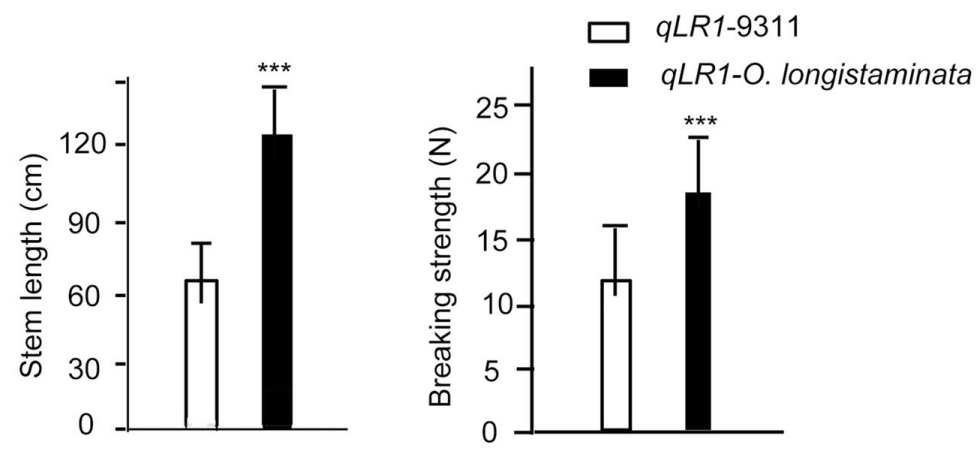

FIGURE 3 | Confirmation of the quantitative trait loci (QTLS) of $q$ LR1. (A) Verification of the qLR1 using seven backcross inbred lines (BILs) to delimit it to an interval between molecular marker between Bin1-161 and Bin1-162. Black rectangle shows the homozygous derived from Oryza longistaminata, white rectangle indicates the homozygous from 93-11. (B). qLR1 effect value analysis of stem diameter, stem length, and breaking strength in O. longistaminata BILs. White shapes mean the alleles from parent 93-11; black shapes indicate the homozygous genotype shared by O. longistaminata.

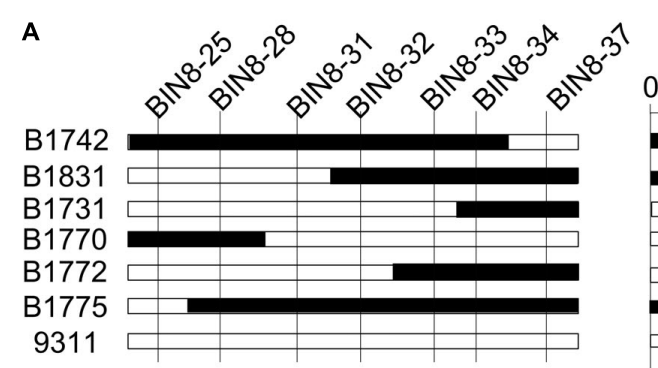

Breaking strength $(\mathrm{N})$

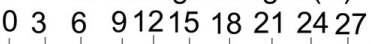

B

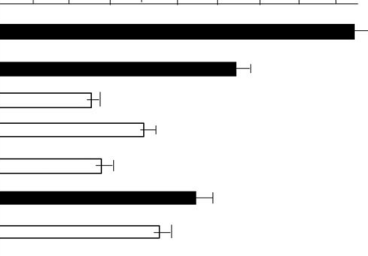

$\square$ qLR8-9311
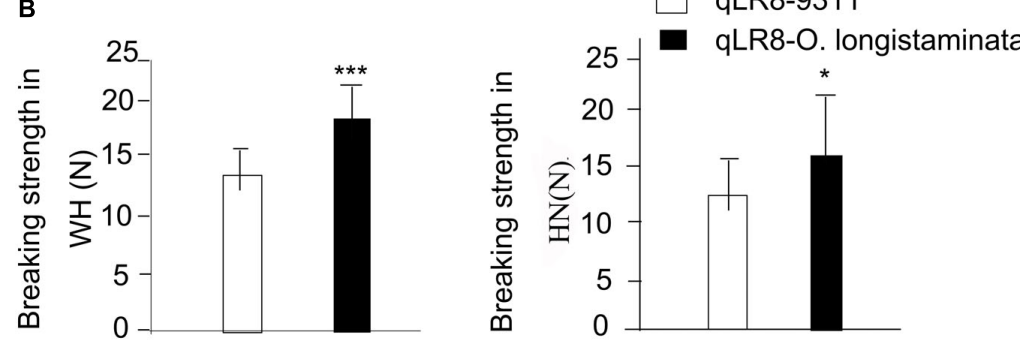

FIGURE 4 | Validation of the quantitative trait loci (QTLs) of $q L R 8$. (A) Verification of the $q L R 8$ using seven backcross inbred lines (BILs) to delimit it to an interval between two marker Bin8-32 and Bin8-33. (B) qLR8 effect value analysis of breaking strength in both Wuhan and Hainan environments. 
Similarly, after high-resolution mapping, we delimited the qLR8 to both tightly linked markers BIN8-32 and BIN8-33 (Figure 4A). Then, we compared the genetic effects of $q L R 8$ on lodging resistance in the BILs. Results showed that the average breaking strength of BILs with $q L R 8$ was $18.89 \mathrm{~N}$ in Hainan, which was significantly higher than that of BILs, which lacked the superior $O$. longistaminata allele at $q L R 8$ with an average breaking strength of $14.34 \mathrm{~N}$ (Figure 4B). These results indicated that $q L R 8$ derived from $O$. longistaminata can significantly increase breaking strength.

To better understand if the two QTLs had additive effects, BIL $1704,1720,1728,1730,1742,1779$, and 1797-2, which carried qLR1 and $q L R 8$, were selected. The breaking strength of the seven lines ranged from 15.92 to $29.68 \mathrm{~N}$, with an average of $22.55 \mathrm{~N}$. This was significantly higher than the average value of the BILs carrying only $q L R 1(19.60 \mathrm{~N})$ and the average value of the BILs carrying only qLR8 (18.89 N) (Supplementary Figure 2). These results indicate that two QTLs can significantly increase the lodging resistance of rice than either one QTL. In summary, $q L R 1$ and $q L R 8$ from $O$. longistaminata can significantly increase the lodging resistance of rice.

\section{DISCUSSION}

Lodging resistance is an important trait that is necessary for achieving high grain yield in rice production (Khush, 1997). In the past 60 years, short plant stature was the major target for improvement of lodging resistance, but many of the dwarfing genes are rarely applied to rice breeding due to deleterious effects on other agronomic traits such as low fertility and bold grains (Wu Z. et al., 2018). The "Green Evolution" gene $s d 1$ is still the only dwarfing source predominantly used to produce semi-dwarf varieties in rice. Recently, some studies have shown the possibility of improving rice yield by increasing plant biomass (Jiang et al., 2016). Increasing plant height is an effective and feasible way to increase biomass from a morphological viewpoint (Donald, 1968; Ying et al., 1998; Ma and Yuan, 2015). In this study, some tall O. longistaminata BILs also exhibited larger stem diameter, more breaking strength, and strong resistance to lodging (Figure 3), indicating that wild rice $O$. longistaminata is a novel genetic resource for breeding strong, lodging-resistant rice.

Although more than 24 QTLs for lodging resistanceassociated traits had been reported earlier with three of them

TABLE 4 | Putative genes at two quantitative trait loci (QTLs) regions for $q L R 1$ and $q L R 8$, associated with lodging resistance in rice.

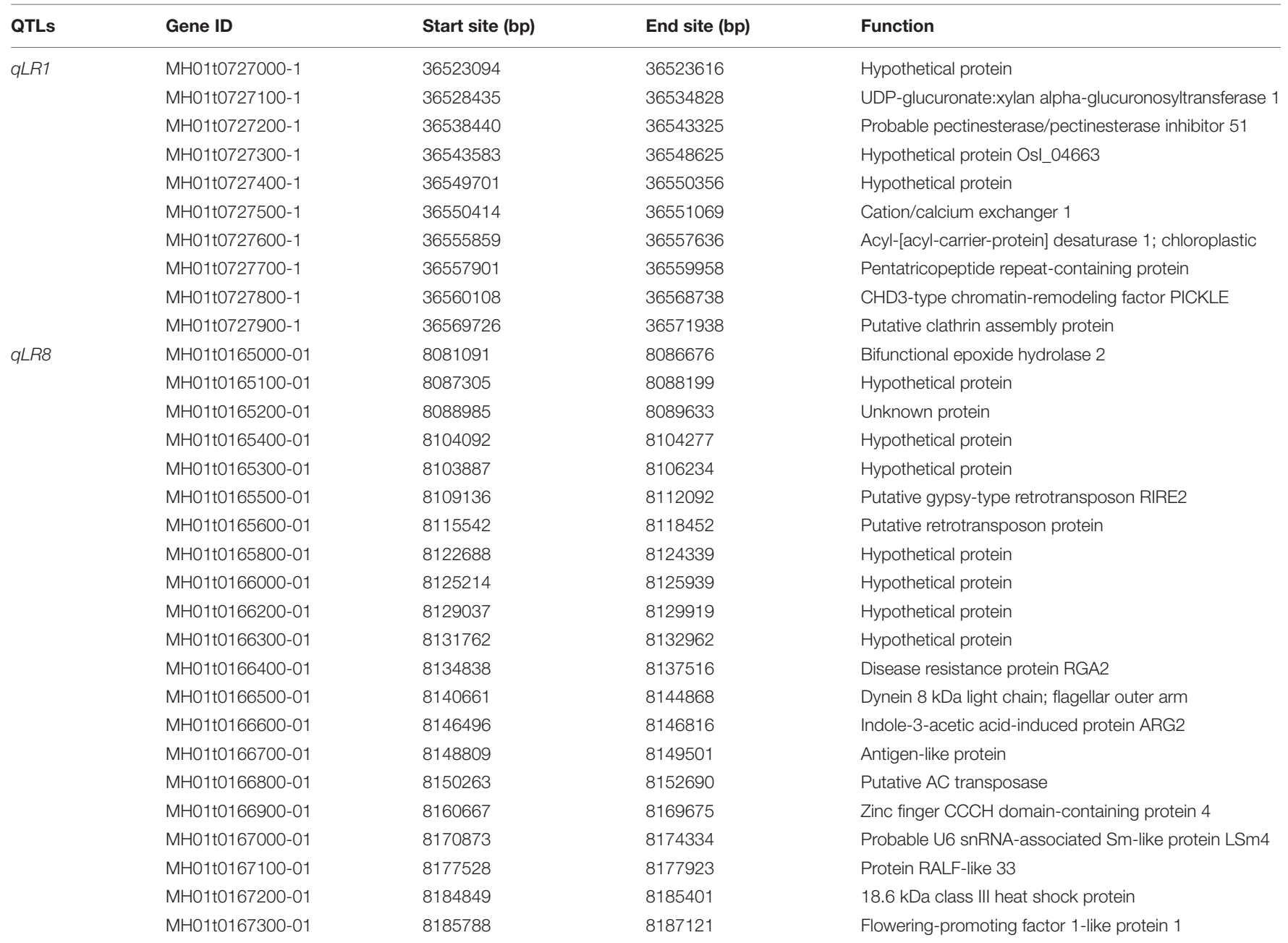


having been cloned in cultivars (Ookawa et al., 2016; Mulsanti et al., 2018; Sowadan et al., 2018), only two major QTLs for lodging resistance had been cloned (Xie et al., 2017; Wu Y. et al., 2018). Gichuhi et al. (2016) had identified three QTLs for culm-base thickness derived from O. longistaminata. None of them was overlapped with our results, which suggests that O. longistaminata contains great potential gene resources for cultivated rice lodging resistance improvement. In this study, we identified 12 new QTLs for lodging resistance with positive alleles derived from wild rice $O$. longistaminata for the first time. In addition, $q L R 1$, a pleiotropic QTL responsible for stem length, stem diameter, and breaking strength, was narrowed to a small region $\sim 0.65 \mathrm{cM}$ covering $\sim 80 \mathrm{~kb}$ physical distance on chromosome 1 (Figure $\mathbf{3 A}$ and Table $\mathbf{3}$ ). Corresponding to the MH63RS1 reference genome ${ }^{2}$, the region contains only 10 predicted genes: two hypothetical proteins and eight functional genes (Table 4). Two genes draw our attention according to their gene annotation. First is MH01t0727100-1 encoding a UDPglucuronate:xylan alpha-glucuronosyltransferase 1, which can enhance mechanical strength of the stem (Gao et al., 2020). Epigenetic plays an increasing important role for plant breeding and selection of adaptive traits. Second is MH01t0727800-1 encoding a CHD3-type chromatin-remodeling factor PICKLE, which is highly associated with variability of growth and gene expression (Zhang et al., 2014). Meanwhile, qLR8, another QTL contributing to stem breaking strength, was localized in an interval of $1.34 \mathrm{cM}$ covering $\sim 120 \mathrm{~kb}$ according on the MH63RS1 genome. This region contained 21 predicted genes: eight hypothetical proteins and 13 functional genes (Table 4). Interestingly, protein RALF-like 33 impacts on acidification and cell expansion during growth and development according to previous report (Murphy and De Smet, 2014). These information will provide insight into further gene cloning combined with the release of the gold $O$. longistaminata's genome. Taken all the identified QTLs into consideration, no QTL for breaking strength was detected from 93-11 (Supplementary Figure 3), which indicates that wild rice $O$. longistaminata can significantly improve the lodging resistance of moderance rice. These QTLs identified in wild rice gave an insight into the genetic basis of lodging resistance.

In rice breeding, lodging resistance is one of the most essential characters required for breeding super high-yielding rice. Grain yield is the product of harvest index (HI) and

${ }^{2}$ http://rice.hzau.edu.cn/rice/

\section{REFERENCES}

Berry, P. M., Sterling, M., Spink, J. H., Baker, C. J., Sylvester-Bradley, R., Mooney, S. J., et al. (2004). Understanding and reducing lodging in cereals. Adv. Agron. 84, 217-271. doi: 10.1007/s11356-016-8237-1

Donald, C. M. (1968). The breeding of crop ideotypes. Euphytica 17, 385-403. doi: $10.1007 / \mathrm{bf} 00056241$

Fan, C., Li, Y., Hu, Z., Hu, H., Wang, G., Li, A., et al. (2018). Ectopic expression of a novel OsExtensin-like gene consistently enhances plant lodging resistance by regulating cell elongation and cell wall thickening in rice. Plant Biotechnol. J. 16, 254-263. doi: $10.1111 /$ pbi.12766 biomass, and previous studies have shown that increasing plant height can increase biomass. Here, two newly identified QTLs, $q L R 1$ and $q L R 8$, both improved stem breaking strength in rice. Furthermore, the former can also improve stem diameter and stem length. These two QTLs do not overlap with any previous reports. It is expected that, when these two QTLs are pyramided together, they can greatly improve lodging resistance in rice. Thus these two QTLs can serve as new resources for breeding superyielding rice varieties.

\section{DATA AVAILABILITY STATEMENT}

The raw data for this study can be found in the BioProject ID PRJNA615752 on NCBI. The URL is https://www.ncbi.nlm.nih. gov/sra/?term=PRJNA615752.

\section{AUTHOR CONTRIBUTIONS}

SL and WL conceived and planned the work. WL, DD, ZY, YC, and WY performed phenotypic screening. SL, NL, and JJ developed the population. WL analyzed the genotypic data. WL, ZZ, and SL drafted the manuscript.

\section{FUNDING}

This work was partly granted from the National Key Research and Development Program (2016YFD0100903), National Transgenic Research Program (2016ZX08001004-001-002), and National Natural Science Foundation (31870322) of China.

\section{ACKNOWLEDGMENTS}

We would like to thank Dr. Xiaoyun Luo for kindly providing the plant lodging tester.

\section{SUPPLEMENTARY MATERIAL}

The Supplementary Material for this article can be found online at: https://www.frontiersin.org/articles/10.3389/fpls.2020.00628/ full\#supplementary-material

Gao, D., Sun, W., Wang, D., Dong, H., Zhang, R., and Yu, S. (2020). A xylan glucuronosyltransferase gene exhibits pleiotropic effects on cellular composition and leaf development in rice. Sci. Rep. 10:3726. doi: 10.1038/ s41598-020-60593-3

Gichuhi, E., Himi, E., Takahashi, H., Zhu, S., Doi, K., Tsugane, K., et al. (2016). Identification of QTLs for yield-related traits in RILs derived from the cross between pLIA-1 carrying Oryza longistaminata chromosome segments and Norin 18 in rice. Breed. Sci. 66, 720-733. doi: 10.1270/jsbbs. 16083

Jiang, P., Xie, X. B., Huang, M., Zhou, X. F., Zhang, R. C., Chen, J. N., et al. (2016). Potential yield increase of hybrid rice at five locations in southern China. Rice 9:11. doi: 10.1186/s12284-016-0085-6 
Jin, J., Long, W., Wang, L., Liu, X., Pan, G., Xiang, W., et al. (2018). QTL mapping of seed vigor of backcross inbred lines derived from oryza longistaminata under artificial aging. Front. Plant Sci. 9:1909. doi: 10.3389/fpls.2018. 01909

Kashiwagi, T., and Ishimaru, K. (2004). Identification and functional analysis of a locus for improvement of lodging resistance in rice. Plant Physiol. 134, 676-683. doi: $10.1104 /$ pp.103.029355

Kashiwagi, T., Madoka, Y., Hirotsu, N., and Ishimaru, K. (2006). Locus prl5 improves lodging resistance of rice by delaying senescence and increasing carbohydrate reaccumulation. Plant Physiol. Biochem. 44, 152-157. doi: 10. 1016/j.plaphy.2006.02.004

Kashiwagi, T., Togawa, E., Hirotsu, N., and Ishimaru, K. (2008). Improvement of lodging resistance with QTLs for stem diameter in rice (Oryza sativa L.). Theor. Appl. Genet. 117, 749-757. doi: 10.1007/s00122-008-0816-1

Khush, G. S. (1997). Origin, dispersal, cultivation and variation of rice. Plant Mol. Biol. 35, 25-34. doi: 10.1007/978-94-011-5794-0_3

Ma, G. H., and Yuan, L. P. (2015). Hybrid rice achievements, development and prospect in China. J. Integrat. Agric. 14, 197-205. doi: 10.16288/j.yczz. 18-213

Meng, L., Li, H., Zhang, L., and Wang, J. (2015). QTL IciMapping: Integrated software for genetic linkage map construction and quantitative trait locus mapping in biparental populations. Crop J. 3, 269-283. doi: 10.1016/j.cj.2015. 01.001

Mulsanti, I. W., Yamamoto, T., Ueda, T., Samadi, A. F., Kamahora, E., Rumanti, I. A., et al. (2018). Finding the superior allele of japonica-type for increasing stem lodging resistance in indica rice varieties using chromosome segment substitution lines. Rice 11:25. doi: 10.1186/s12284-018-0216-3

Murai, M., Takamure, I., Sato, S., Tokutome, T., and Sato, Y. (2002). Effects of the dwarfing gene originating from 'Dee-geo-woo-gen' on yield and its related traits in rice. Breed. Sci. 52, 95-100. doi: 10.1270/jsbbs.52.95

Murphy, E., and De Smet, I. (2014). Understanding the RALF family: a tale of many species. Trends Plant Sci. 19, 664-671. doi: 10.1016/j.tplants.2014.06.005

Okuno, A., Hirano, K., Asano, K., Takase, W., Masuda, R., Morinaka, Y., et al. (2014). New approach to increasing rice lodging resistance and biomass yield through the use of high gibberellin producing varieties. PLoS One 9:e86870. doi: 10.1371 /journal.pone.0086870

Ookawa, T., Aoba, R., Yamamoto, T., Ueda, T., Takai, T., Fukuoka, S., et al. (2016). Precise estimation of genomic regions controlling lodging resistance using a set of reciprocal chromosome segment substitution lines in rice. Sci. Rep. 6:30572. doi: 10.1038/srep30572

Ookawa, T., Hobo, T., Yano, M., Murata, K., Ando, T., Miura, H., et al. (2010). New approach for rice improvement using a pleiotropic QTL gene for lodging resistance and yield. Nat. Commun. 1:132.

Peng, D. L., Chen, X. G., Yin, Y. P., Lu, K. L., Yang, W. B., Tang, Y. H., et al. (2014). Lodging resistance of winter wheat (Triticum aestivum L.): lignin accumulation and its related enzymes activities due to the application of paclobutrazol or gibberellin acid. Field Crops Res. 157, 1-7. doi: 10.1016/j.fcr.2013. 11.015
Peng, J., Richards, D. E., Hartley, N. M., Murphy, G. P., Devos, K. M., Flintham, J. E., et al. (1999). 'Green revolution' genes encode mutant gibberellin response modulators. Nature 400, 256-261. doi: 10.1038/22307

Sowadan, O., Li, D. L., Zhang, Y. Q., Zhu, S. S., Hu, X. X., Bhanbhro, L. B., et al. (2018). Mining of favorable alleles for lodging resistance traits in rice (Oryza sativa) through association mapping. Planta 248, 155-169. doi: 10.1007/ s00425-018-2885-y

Wu, Y., Zhao, S., Li, X., Zhang, B., Jiang, L., Tang, Y., et al. (2018). Deletions linked to PROG1 gene participate in plant architecture domestication in Asian and African rice. Nat. Commun. 9:4157. doi: 10.1038/s41467-01806509-2

Wu, Z., Tang, D., Liu, K., Miao, C., Zhuo, X., Li, Y., et al. (2018). Characterization of a new semi-dominant dwarf allele of SLR1 and its potential application in hybrid rice breeding. J. Exp. Bot. 69, 4703-4713. doi: 10.1093/jxb/ery243

Xie, Y. Y., Xu, P., Huang, J. L., Ma, S. J., Xie, X. R., Tao, D. Y., et al. (2017). Interspecific hybrid sterility in rice is mediated by OgTPR1 at the S1 Locus encoding a peptidase-like protein. Mol. Plant 10, 1137-1140. doi: 10.1016/j. molp.2017.05.005

Yadav, S., Singh, U. M., Naik, S. M., Venkateshwarlu, C., Ramayya, P. J., Raman, K. A., et al. (2017). Molecular mapping of QTLs associated with lodging resistance in dry direct-seeded rice (Oryza sativa L.). Front. Plant Sci. 8:1431. doi: 10.3389/fpls.2017.01431

Ying, J. F., Peng, S. B., He, Q. R., Yang, H., Yang, C. D., Visperas, R. M., et al. (1998). Comparison of high-yield rice in tropical and subtropical environments - I. Determinants of grain and dry matter yields. Field Crops Res. 57, 71-84. doi: 10.1016/s0378-4290(98)00077-x

Yuan, L. P. (2017). Progress in super-hybrid rice breeding. Crop J. 5, 100-102. doi: 10.1038/s41598-017-18576-4

Zhang, D., Jing, Y., Jiang, Z., and Lin, R. (2014). The chromatin-remodeling factor PICKLE integrates brassinosteroid and gibberellin signaling during skotomorphogenic growth in Arabidopsis. Plant Cell 26, 2472-2485. doi: 10. 1105/tpc.113.121848

Zhu, L. Y., Chen, J. T., Li, D., Zhang, J. H., Huang, Y. Q., Zhao, Y. F., et al. (2013). QTL mapping for stalk related traits in maize (Zea mays L.) Under different densities. J. Integrat. Agric. 12, 218-228. doi: 10.1016/s2095-3119(13) 60221-x

Conflict of Interest: The authors declare that the research was conducted in the absence of any commercial or financial relationships that could be construed as a potential conflict of interest.

Copyright (c) 2020 Long, Dan, Yuan, Chen, Jin, Yang, Zhang, Li and Li. This is an open-access article distributed under the terms of the Creative Commons Attribution License (CC BY). The use, distribution or reproduction in other forums is permitted, provided the original author(s) and the copyright owner(s) are credited and that the original publication in this journal is cited, in accordance with accepted academic practice. No use, distribution or reproduction is permitted which does not comply with these terms. 